\title{
Quenching time for a system of semilinear heat equations
}

Theodore K. Boni, Halima Nachid, and Diabate Nabongo 


\title{
QUENCHING TIME FOR A SYSTEM OF SEMILINEAR HEAT EQUATIONS
}

\author{
THEODORE K. BONI, HALIMA NACHID, AND DIABATE NABONGO
}

Received 21 June, 2008

\begin{abstract}
This paper concerns the study of the quenching time of the solution of the initialboundary value problem for a system of reaction-diffusion equations.
\end{abstract}

2000 Mathematics Subject Classification: 35B40, 35K50, 65M06

Keywords: quenching, semilinear parabolic system, numerical quenching time

\section{INTRODUCTION}

Let $\Omega$ be a bounded domain in $\mathbb{R}^{N}$ with the smooth boundary $\partial \Omega$. Consider the following initial-boundary value problem for a system of reaction-diffusion equations of the form

$$
\begin{gathered}
u_{t}=\varepsilon \Delta u+f(v) \quad \text { in } \quad \Omega \times(0, T), \\
v_{t}=\varepsilon \Delta v+g(u) \quad \text { in } \quad \Omega \times(0, T), \\
u=0 \quad \text { on } \quad \partial \Omega \times(0, T), \\
v=0 \quad \text { on } \quad \partial \Omega \times(0, T), \\
u(x, 0)=u_{0}(x) \geq 0 \quad \text { in } \quad \Omega, \\
v(x, 0)=v_{0}(x) \geq 0 \quad \text { in } \quad \Omega,
\end{gathered}
$$

where $f:\left(-\infty, b_{1}\right) \rightarrow(0, \infty)$ is $C^{1}$ convex, increasing function with $b_{1}=$ const $>0$, $\lim _{s \rightarrow b_{1}} f(s)=+\infty, \int_{0}^{b_{1}} \frac{d s}{f(s)}<+\infty, g:\left(-\infty, b_{2}\right) \rightarrow(0, \infty)$ is $C^{1}$ convex, increasing function with $b_{2}=$ const $>0, \lim _{s \rightarrow b_{2}} g(s)=+\infty, \int_{0}^{b_{2}} \frac{d s}{g(s)}<+\infty$. The initial data $\left(u_{0}, v_{0}\right)$ is such that $u_{0} \in C^{1}(\bar{\Omega}), v_{0} \in C^{1}(\bar{\Omega}), u_{0}(x) \geq 0$ in $\Omega, u_{0}(x)=0$ on $\partial \Omega, v_{0}(x) \geq 0$ in $\Omega, v_{0}(x)=0$ on $\partial \Omega, \sup _{x \in \Omega} u_{0}(x)<b_{2}, \sup _{x \in \Omega} v_{0}(x)<b_{1}$. Here, $(0, T)$ is the maximal time interval of existence of the solution $(u, v)$. The time $T$ may be finite or infinite. When $T$ is infinite, then we say that the solution $u$ exists globally. When $T$ is finite, then the solution $(u, v)$ develops a singularity in a finite 
time, namely

$$
\left(\lim _{t \rightarrow T}\|u(\cdot, t)\|_{\infty}, \lim _{t \rightarrow T}\|v(\cdot, t)\|_{\infty}\right) \not \leq\left(b_{2}, b_{1}\right),
$$

where $\|u(\cdot, t)\|_{\infty}=\max _{x \in \Omega}|u(x, t)|$. Here, $(a, b) \not \leq(c, d)$ means that $(a, b) \leq(c, d)$ and at least one of the equalities $a=c, b=d$ is valid.

Solutions for systems of semilinear heat equations which quench in a finite time have been the subject of investigation of several authors (see [3-5] and the references cited therein). By standard methods based on the maximum principle, the local existence, uniqueness, global existence, and quenching have been treated. One may also find in $[1,6,7,9,10]$ some results about quenching for semilinear heat equations. In this paper, we are interested in the asymptotic behaviour of the quenching time as $\varepsilon$ goes to zero. Our work is motivated by the paper [8] by Friedman and Lacey, where they consider the initial-boundary value problem

$$
\begin{gathered}
u_{t}=\varepsilon \Delta u+g(u) \quad \text { in } \Omega \times(0, T), \\
u=0 \quad \text { on } \partial \Omega \times(0, T), \\
u(x, 0)=u_{0}(x) \geq 0 \quad \text { in } \Omega,
\end{gathered}
$$

where $g(s)$ is a positive, increasing, convex function for the nonnegative values of $s$ and such that $\int_{0}^{+\infty} \frac{d s}{g(s)}<+\infty$. The initial data $u_{0}$ is a positive and continuous function in $\bar{\Omega}$. Under some additional conditions on the initial data, they showed that the solution $u$ of (1.7)-(1.9) blows up in a finite time, and its blow-up time goes to that of the solution $\alpha(t)$ of the

$$
\alpha^{\prime}(t)=g(\alpha(t)), \quad t>0, \quad \alpha(0)=M,
$$

when $\varepsilon$ goes to zero, where $M=\sup _{x \in \Omega} u_{0}(x)$ (we say that a solution $u$ blows up in a finite time if it reaches the value infinity in a finite time). Nabongo and Boni have obtained in [10] an analogous result in the case of the phenomenon of quenching for semilinear heat equations. In this article, we get a comparable result for the system described in (1.1)-(1.6). More precisely, under some hypotheses, we show that if $\varepsilon$ is small enough, then the solution $(u, v)$ of $(1.1)-(1.6)$ quenches in a finite time, and its quenching time tends to that of the solution $(\alpha(t), \beta(t))$ of the differential system defined below

$$
\begin{gathered}
\alpha^{\prime}(t)=f(\beta(t)), \quad t>0, \\
\beta^{\prime}(t)=g(\alpha(t)), \quad t>0, \\
\alpha(0)=M_{1}, \\
\beta(0)=M_{2},
\end{gathered}
$$

as $\varepsilon$ goes to zero, where $M_{1}=\sup _{x \in \Omega} u_{0}(x), M_{2}=\sup _{x \in \Omega} v_{0}(x)$.

Our paper is written in the following manner. In the next section, under some conditions, we prove that if $\varepsilon$ is small enough, then the solution $(u, v)$ of $(1.1)-(1.6)$ 
quenches in a finite time, and its quenching time tends to that of the solution of the differential system defined in (1.11)-(1.14). Finally, in the last section, we give some numerical results to illustrate our analysis.

\section{QUENCHING SOLUTIONS}

In this section, under some assumptions, we prove that if $\varepsilon$ is small enough, then the solution $(u, v)$ of (1.1)-(1.6) quenches in a finite time, and its quenching time goes to that of the solution of the differential system defined in (1.11)-(1.14) as $\varepsilon$ goes to zero. We start by recalling an important result.

Consider the following eigenvalue problem

$$
\begin{gathered}
-\Delta \varphi(x)=\lambda \varphi(x) \quad \text { in } \Omega, \\
\varphi(x)=0 \quad \text { on } \quad \partial \Omega, \\
\varphi(x)>0 \text { in } \Omega .
\end{gathered}
$$

It is well known that the above eigenvalue problem admits a solution $(\varphi, \lambda)$ such that $\lambda>0$. We can normalize $\varphi$ so that $\int_{\Omega} \varphi(x) d x=1$. Now, let us give our first result about the quenching time.

Theorem 1. Suppose that $u_{0}(x)=0$ and $v_{0}(x)=0$. Let

$$
A=\lambda \max \left\{\frac{b_{2}}{f(0)}, \frac{b_{1}}{g(0)}\right\} .
$$

If $\varepsilon<\frac{1}{A}$, then the solution $(u, v)$ of (1.1)-(1.6) quenches in a finite time, and its quenching time $T$ obeys the following estimates

$$
0 \leq T-T_{0} \leq \varepsilon T_{0} A+o(\varepsilon),
$$

where $T_{0}$ is the quenching time of the solution $(\alpha(t), \beta(t))$ of the differential system defined in (1.11)-(1.14).

Proof. Since $(0, T)$ is the maximal time interval of existence of the solution $(u, v)$, our aim is to show that $T$ is finite and satisfies the above estimates. Introduce the functions $w(t)$ and $z(t)$ defined as follows

$$
w(t)=\int_{\Omega} u(x, t) \varphi(x) d x, \quad z(t)=\int_{\Omega} v(x, t) \varphi(x) d x, \quad t \in[0, T) .
$$

Due to the fact that the initial data $\left(u_{0}, v_{0}\right)$ is nonnegative in $\Omega$, from the maximum principle, $(u, v)$ is also nonnegative in $\Omega \times(0, T)$. Take the derivative of $w$ in $t$ and use (1.1) to obtain

$$
w^{\prime}(t)=\varepsilon \int_{\Omega} \varphi \Delta u d x+\int_{\Omega} f(v) \varphi d x \quad \text { for } t \in(0, T) .
$$


Applying Green's formula, we arrive at the equality

$$
w^{\prime}(t)=\varepsilon \int_{\Omega} u \Delta \varphi d x+\int_{\Omega} f(v) \varphi d x \quad \text { for } t \in(0, T) .
$$

It follows from (2.1) and Jensen's inequality that

$$
w^{\prime}(t) \geq-\varepsilon \lambda w(t)+f(z(t)) \quad \text { for } t \in(0, T) .
$$

In the same way, we also get

$$
z^{\prime}(t) \geq-\varepsilon \lambda z(t)+g(w(t)) \quad \text { for } t \in(0, T) .
$$

It is not difficult to see that $0 \leq w(t) \leq b_{2}$ and $0 \leq z(t) \leq b_{1}$. We deduce that

$$
\begin{array}{ll}
w^{\prime}(t) \geq-\varepsilon \lambda b_{2}+f(z(t)) & \text { for } t \in(0, T), \\
z^{\prime}(t) \geq-\varepsilon \lambda b_{1}+g(w(t)) & \text { for } t \in(0, T),
\end{array}
$$

whence

$$
\begin{array}{ll}
w^{\prime}(t) \geq f(z(t))\left(1-\frac{\varepsilon \lambda b_{2}}{f(z(t))}\right) & \text { for } t \in(0, T), \\
z^{\prime}(t) \geq g(w(t))\left(1-\frac{\varepsilon \lambda b_{1}}{g(w(t))}\right) & \text { for } t \in(0, T) .
\end{array}
$$

Since $f(z(t)) \geq f(0)$ and $g(w(t)) \geq g(0)$, we arrive at the inequalities

$$
\begin{array}{cc}
w^{\prime}(t) \geq f(z(t))\left(1-\frac{\varepsilon \lambda b_{2}}{f(0)}\right) & \text { for } t \in(0, T), \\
z^{\prime}(t) \geq g(w(t))\left(1-\frac{\varepsilon \lambda b_{1}}{g(0)}\right) & \text { for } t \in(0, T) .
\end{array}
$$

Taking into account the fact that $\frac{\lambda b_{2}}{f(0)} \leq A$ and $\frac{\lambda b_{1}}{g(0)} \leq A$, we find that

$$
\begin{array}{ll}
w^{\prime}(t) \geq f(z(t))(1-\varepsilon A) & \text { for } t \in(0, T), \\
z^{\prime}(t) \geq g(w(t))(1-\varepsilon A) & \text { for } t \in(0, T) .
\end{array}
$$

Let us set

$$
\begin{aligned}
& w_{1}(t)=w\left(\frac{t}{1-\varepsilon A}\right) \quad \text { for } t \in(0,(1-\varepsilon A) T), \\
& z_{1}(t)=z\left(\frac{t}{1-\varepsilon A}\right) \quad \text { for } t \in(0,(1-\varepsilon A) T) .
\end{aligned}
$$

A straightforward computation reveals that

$$
\begin{array}{lll}
w_{1}^{\prime}(t) \geq f\left(z_{1}(t)\right) & \text { for } t \in(0,(1-\varepsilon A) T), & w_{1}(0)=w(0)=0, \\
z_{1}^{\prime}(t) \geq g\left(w_{1}(t)\right) & \text { for } t \in(0,(1-\varepsilon A) T), & z_{1}(0)=z(0)=0 .
\end{array}
$$

From the maximum principle, we get

$$
w_{1}(t) \geq \alpha(t) \text { for } t \in\left(0, T_{*}\right),
$$




$$
z_{1}(t) \geq \beta(t) \text { for } t \in\left(0, T_{*}\right),
$$

where $T_{*}=\min \left\{T_{0},(1-\varepsilon A) T\right\}$, which implies that

$$
T \leq \frac{T_{0}}{1-\varepsilon A} .
$$

In fact, suppose that $T>\frac{T_{0}}{1-\varepsilon A}=T^{\prime}$. We deduce that

$$
\left(w\left(T^{\prime}\right), z\left(T^{\prime}\right)\right)=\left(w_{1}\left(T_{0}\right), z_{1}\left(T_{0}\right)\right) \geq\left(\alpha\left(T_{0}\right), \beta\left(T_{0}\right)\right) \not \leq\left(b_{2}, b_{1}\right) .
$$

Since

$$
\left(\left\|u\left(\cdot, T^{\prime}\right)\right\|_{\infty},\left\|v\left(\cdot, T^{\prime}\right)\right\|_{\infty}\right) \geq\left(w\left(T^{\prime}\right), z\left(T^{\prime}\right)\right),
$$

we have a contradiction because $(0, T)$ is the maximum time interval of existence of $(u, v)$. On the other hand, let $\left(u_{1}(x, t), v_{1}(x, t)\right)$ be such that

$$
\begin{array}{ll}
u_{1}(x, t)=\alpha(t) & \text { in } \quad \bar{\Omega} \times\left[0, T_{0}\right), \\
v_{1}(x, t)=\beta(t) & \text { in } \quad \bar{\Omega} \times\left[0, T_{0}\right) .
\end{array}
$$

A routine computation reveals that

$$
\begin{array}{ccc}
u_{1 t}=\varepsilon \Delta u_{1}+f\left(v_{1}\right) & \text { in } & \Omega \times\left(0, T_{0}\right), \\
v_{1 t}=\varepsilon \Delta v_{1}+g\left(u_{1}\right) & \text { in } & \Omega \times\left(0, T_{0}\right),
\end{array}
$$

$u_{1} \geq 0$ on $\partial \Omega \times\left(0, T_{0}\right), v_{1} \geq 0$ on $\partial \Omega \times\left(0, T_{0}\right)$, and

$$
\begin{array}{ll}
u_{1}(x, 0) \geq 0 \quad \text { in } \quad & \Omega, \\
v_{1}(x, 0) \geq 0 \quad \text { in } \quad \Omega .
\end{array}
$$

The maximum principle implies that

$$
\begin{array}{lll}
0 \leq u(x, t) \leq u_{1}(x, t)=\alpha(t) & \text { in } & \Omega \times\left(0, T^{0}\right), \\
0 \leq v(x, t) \leq v_{1}(x, t)=\beta(t) & \text { in } & \Omega \times\left(0, T^{0}\right),
\end{array}
$$

where $T^{0}=\min \left\{T, T_{0}\right\}$. We deduce that

$$
T \geq T_{0} .
$$

Indeed, assume that $T<T_{0}$. We get

$$
\left(\|u(\cdot, T)\|_{\infty},\|v(\cdot, T)\|_{\infty}\right) \leq(\alpha(T), \beta(T))<\left(b_{2}, b_{1}\right),
$$

which is a contradiction because $(0, T)$ is the maximal time interval of existence of the solution $(u, v)$. Applying Taylor's expansion, we obtain

$$
\frac{1}{1-\varepsilon A}=1+\varepsilon A+o(\varepsilon) \text {. }
$$

Using (2.4), (2.5) and the relation above, we complete the proof. 
Now, let us consider the case where the initial data is not null. In the sequel, we suppose that there exists $a \in \Omega$ such that

$$
\sup _{x \in \Omega} u_{0}(x)=u_{0}(a) \text { and } \sup _{x \in \Omega} v_{0}(x)=v_{0}(a) .
$$

Consider the following eigenvalue problem

$$
\begin{gathered}
-\Delta \psi(x)=\lambda_{\delta} \psi(x) \quad \text { in } \quad B(a, \delta), \\
\psi(x)=0 \quad \text { on } \quad \partial B(a, \delta), \\
\psi(x)>0 \quad \text { in } \quad B(a, \delta),
\end{gathered}
$$

where $\delta>0$, such that, $B(a, \delta)=\left\{x \in \mathbb{R}^{N} ;\|x-a\|<\delta\right\} \subset \Omega$.

It is well known that the above problem has a solution $\left(\psi, \lambda_{\delta}\right)$ such that $\lambda_{\delta}=\frac{\lambda_{1}}{\delta^{2}}$, where $\lambda_{1}$ is the eigenvalue corresponding to the above eigenvalue problem for $\delta=1$.

We are in position to state our result in the case where the initial data is not null.

Theorem 2. Suppose that $M_{1}=\sup _{x \in \Omega} u_{0}(x)>0, M_{2}=\sup _{x \in \Omega} v_{0}(x)>0$, and let $K$ be an upper bound of the first derivatives of $u_{0}$ and $v_{0}$. Let

If

$$
A=\lambda_{1} \max \left\{\frac{K^{2} b_{2}}{f(0)}, \frac{K^{2} b_{1}}{g(0)}\right\} .
$$

$$
\varepsilon<\min \left\{\left(M_{1} / 2\right)^{3},\left(M_{2} / 2\right)^{3},(2 A)^{-3},(K \operatorname{dist}(a, \partial \Omega))^{3}\right\},
$$

then the solution $(u, v)$ of (1.1)-(1.6) quenches in a finite time, and its quenching time $T$ obeys the estimates

$$
0 \leq T-T_{0} \leq\left(T_{0} A+C\right) \varepsilon^{1 / 3}+o\left(\varepsilon^{1 / 3}\right),
$$

where

$$
C=\frac{1}{\min \left\{\frac{1}{2} f\left(\frac{M_{2}}{2}\right), \frac{1}{2} g\left(\frac{M_{1}}{2}\right)\right\}}
$$

and $T_{0}$ is the quenching time of the solution $(\alpha(t), \beta(t))$ of the differential system defined in (1.11)-(1.14).

Proof. Due to the fact that $u_{0} \in C^{1}(\bar{\Omega})$ and $v_{0} \in C^{1}(\bar{\Omega})$, using the mean value theorem and the triangle inequality, we get

$$
\begin{array}{ll}
u_{0}(x) \geq u_{0}(a)-\varepsilon^{1 / 3} & \text { for } x \in B(a, \delta) \subset \Omega, \\
v_{0}(x) \geq v_{0}(a)-\varepsilon^{1 / 3} & \text { for } x \in B(a, \delta) \subset \Omega,
\end{array}
$$

where $\delta=\frac{\varepsilon^{1 / 3}}{K}$. Since the initial data $\left(u_{0}, v_{0}\right)$ is nonnegative in $\Omega$, from the maximum principle, $(u, v)$ is also nonnegative in $\Omega \times(0, T)$. Introduce the functions $w(t)$ and $z(t)$ defined as follows

$$
w(t)=\int_{B(a, \delta)} u(x, t) \psi(x) d x, \quad z(t)=\int_{B(a, \delta)} v(x, t) \psi(x) d x, \quad t \in[0, T) .
$$


Take the derivative of $w$ in $t$ and use (1.1) to obtain

$$
w^{\prime}(t)=\varepsilon \int_{B(a, \delta)} \psi \Delta u d x+\int_{B(a, \delta)} f(v) \psi d x \quad \text { for } t \in(0, T) .
$$

Applying Green's formula, we arrive at

$$
\begin{aligned}
w^{\prime}(t)= & \varepsilon \int_{B(a, \delta)} u \Delta \psi d x-\varepsilon \int_{\partial B(a, \delta)} u \frac{\partial \psi}{\partial \nu} d s+\varepsilon \int_{\partial B(a, \delta)} \psi \frac{\partial u}{\partial \nu} d s \\
& +\int_{B(a, \delta)} f(v) \psi d x \quad \text { for } t \in(0, T),
\end{aligned}
$$

where $v$ is the exterior normal unit vector on $\partial B(a, \delta)$. Taking into account (2.6), (2.7) and the fact that $\frac{\partial \psi}{\partial v} \leq 0$, we arrive at the relation

$$
w^{\prime}(t) \geq-\varepsilon \lambda_{\delta} w(t)+\int_{B(a, \delta)} f(v) \psi d x \quad \text { for } t \in(0, T) .
$$

It follows from Jensen's inequality that

$$
w^{\prime}(t) \geq-\varepsilon \lambda_{\delta} w(t)+f(z(t)) \text { for } t \in(0, T) .
$$

In the same way, we also prove that

$$
z^{\prime}(t) \geq-\varepsilon \lambda_{\delta} z(t)+g(w(t)) \text { for } t \in(0, T) .
$$

As in the proof of Theorem 1, we get

$$
\begin{array}{ll}
w^{\prime}(t) \geq f(z(t))\left(1-\frac{\varepsilon \lambda_{\delta} b_{2}}{f(0)}\right) & \text { for } t \in(0, T), \\
z^{\prime}(t) \geq g(w(t))\left(1-\frac{\varepsilon \lambda_{\delta} b_{1}}{g(0)}\right) & \text { for } t \in(0, T),
\end{array}
$$

which implies that

$$
\begin{array}{ll}
w^{\prime}(t) \geq f(z(t))\left(1-\frac{\varepsilon^{1 / 3} \lambda_{1} K^{2} b_{2}}{f(0)}\right) & \text { for } t \in(0, T), \\
z^{\prime}(t) \geq g(w(t))\left(1-\frac{\varepsilon^{1 / 3} \lambda_{1} K^{2} b_{1}}{g(0)}\right) & \text { for } t \in(0, T),
\end{array}
$$

because $\lambda_{\delta}=\frac{\lambda_{1}}{\delta^{2}}=\frac{\lambda_{1} K^{2}}{\varepsilon^{2 / 3}}$. Consequently,

$$
\begin{aligned}
& w^{\prime}(t) \geq f(z(t))\left(1-\varepsilon^{1 / 3} A\right) \quad \text { for } t \in(0, T), \\
& w(0) \geq M_{1}-\varepsilon^{1 / 3},
\end{aligned}
$$

and

$$
z^{\prime}(t) \geq g(w(t))\left(1-\varepsilon^{1 / 3} A\right) \quad \text { for } t \in(0, T),
$$




$$
z(0) \geq M_{2}-\varepsilon^{1 / 3} .
$$

We have

$$
w^{\prime}(t) \geq f(z(0))\left(1-\varepsilon^{1 / 3} A\right) \geq \frac{1}{2} f\left(M_{2} / 2\right)
$$

for $t \in(0, T)$. In the same way, we get $z^{\prime}(t) \geq \frac{1}{2} g\left(M_{1} / 2\right)$ for $t \in(0, T)$, which implies that

$$
w^{\prime}(t) \geq \frac{1}{C}, \quad z^{\prime}(t) \geq \frac{1}{C}
$$

for $t \in(0, T)$. Using the mean value theorem, we get

$$
w\left(C \varepsilon^{1 / 3}\right) \geq M_{1}, \quad z\left(C \varepsilon^{1 / 3}\right) \geq M_{2} .
$$

Set

$$
w_{1}(t)=w\left(\frac{t}{1-\varepsilon^{1 / 3} A}+C \varepsilon^{1 / 3}\right)
$$

for $t \in\left(0,\left(T-C \varepsilon^{1 / 3}\right)\left(1-\varepsilon^{1 / 3} A\right)\right)$, and

$$
z_{1}(t)=z\left(\frac{t}{1-\varepsilon^{1 / 3} A}+C \varepsilon^{1 / 3}\right)
$$

for $t \in\left(0,\left(T-C \varepsilon^{1 / 3}\right)\left(1-\varepsilon^{1 / 3} A\right)\right)$. A straightforward computation reveals that

$$
\begin{aligned}
& w_{1}^{\prime}(t) \geq f\left(z_{1}(t)\right) \quad \text { for } t \in\left(0,\left(T-C \varepsilon^{1 / 3}\right)\left(1-\varepsilon^{1 / 3} A\right)\right), \\
& w_{1}(0) \geq M_{1}
\end{aligned}
$$

and

$$
\begin{aligned}
& z_{1}^{\prime}(t) \geq g\left(w_{1}(t)\right) \quad \text { for } t \in\left(0,\left(T-C \varepsilon^{1 / 3}\right)\left(1-\varepsilon^{1 / 3} A\right)\right), \\
& z_{1}(0) \geq M_{2} .
\end{aligned}
$$

It follows from the maximum principle that

$$
\begin{array}{ll}
w_{1}(t) \geq \alpha(t) & \text { for } t \in\left(0, T^{*}\right), \\
z_{1}(t) \geq \beta(t) & \text { for } t \in\left(0, T^{*}\right),
\end{array}
$$

where $T^{*}=\min \left\{T_{0},\left(T-C \varepsilon^{1 / 3}\right)\left(1-\varepsilon^{1 / 3} A\right)\right\}$. We deduce that

$$
T \leq \frac{T_{0}}{1-\varepsilon^{1 / 3} A}+C \varepsilon^{1 / 3} \text {. }
$$

Indeed, suppose that

We get

$$
T>\frac{T_{0}}{1-\varepsilon^{1 / 3} A}+C \varepsilon^{1 / 3}=T^{\prime}
$$

$$
\left(w\left(T^{\prime}\right), z\left(T^{\prime}\right)\right)=\left(w_{1}\left(T_{0}\right), z_{1}\left(T_{0}\right)\right) \geq\left(\alpha\left(T_{0}\right), \beta\left(T_{0}\right)\right) \not \leq\left(b_{2}, b_{1}\right) .
$$


Since $\left(\left\|u\left(\cdot, T^{\prime}\right)\right\|_{\infty},\left\|v\left(\cdot, T^{\prime}\right)\right\|_{\infty}\right) \geq\left(w\left(T^{\prime}\right), z\left(T^{\prime}\right)\right)$, we have a contradiction because $(0, T)$ is the maximal time interval of existence of the solution $(u, v)$. On the other hand, setting

$$
\begin{array}{ll}
w_{2}(x, t)=\alpha(t) \quad \text { in } & \bar{\Omega} \times\left[0, T_{0}\right), \\
z_{2}(x, t)=\beta(t) & \text { in } \quad \bar{\Omega} \times\left[0, T_{0}\right),
\end{array}
$$

it is not difficult to see that

$$
\begin{gathered}
\left(w_{2}\right)_{t}=\varepsilon \Delta w_{2}+f\left(z_{2}\right) \quad \text { in } \quad \Omega \times\left(0, T_{0}\right), \\
\left(z_{2}\right)_{t}=\varepsilon \Delta z_{2}+g\left(w_{2}\right) \quad \text { in } \Omega \times\left(0, T_{0}\right), \\
w_{2} \geq 0 \quad \text { on } \quad \partial \Omega \times\left(0, T_{0}\right), \\
z_{2} \geq 0 \quad \text { on } \quad \partial \Omega \times\left(0, T_{0}\right), \\
w_{2}(x, 0) \geq u_{0}(x) \quad \text { in } \Omega, \\
z_{2}(x, 0) \geq v_{0}(x) \quad \text { in } \Omega .
\end{gathered}
$$

It follows from the maximum principle that

$$
\begin{aligned}
& \alpha(t)=w_{2}(x, t) \geq u(x, t) \quad \text { in } \quad \Omega \times\left(0, T_{*}^{*}\right), \\
& \beta(t)=z_{2}(x, t) \geq v(x, t) \quad \text { in } \quad \Omega \times\left(0, T_{*}^{*}\right),
\end{aligned}
$$

where $T_{*}^{*}=\min \left\{T_{0}, T\right\}$. We deduce that

$$
T \geq T_{0} \text {. }
$$

Indeed, suppose that $T<T_{0}$. We get

$$
\left(\|u(\cdot, T)\|_{\infty},\|v(\cdot, T)\|_{\infty}\right) \leq(\alpha(T), \beta(T))<\left(b_{2}, b_{1}\right),
$$

which is a contradiction because $(0, T)$ is the maximal time interval of existence of the solution $(u, v)$. Applying Taylor's expansion, we obtain

$$
\frac{1}{1-\varepsilon^{1 / 3} A}=1+\varepsilon^{1 / 3}+o\left(\varepsilon^{1 / 3}\right) .
$$

Using (2.9), (2.10) and the above relation, we complete the proof.

\section{NUMERICAL EXPERIMENTS}

In this section, we give some computational results to confirm the theory established in the previous section. We consider the radial symmetric solution of the problem (1.1)-(1.6) in the case where $\Omega=B=\left\{x \in \mathbb{R}^{N} ;\|x\|<1\right\}, \partial \Omega=S=\{x \in$ $\left.\mathbb{R}^{N} ;\|x\|=1\right\}, f(v)=(1-v)^{-p}, g(u)=(2-u)^{-q}$ with $p>0, q>0$. The above problem may be rewritten in the following form

$$
\begin{array}{ll}
u_{t}=\varepsilon\left(u_{r r}+\frac{N-1}{r} u_{r}\right)+(1-v)^{-p}, & r \in(0,1), \quad t \in(0, T), \\
v_{t}=\varepsilon\left(v_{r r}+\frac{N-1}{r} v_{r}\right)+(2-u)^{-q}, & r \in(0,1), \quad t \in(0, T),
\end{array}
$$




$$
\begin{gathered}
u_{r}(0, t)=0, \quad u(1, t)=0, \quad t \in(0, T), \\
v_{r}(0, t)=0, \quad v(1, t)=0, \quad t \in(0, T), \\
u(r, 0)=\varphi(r), \quad r \in(0,1), \\
v(r, 0)=\psi(r), \quad r \in(0,1) .
\end{gathered}
$$

Here, we take

$$
\begin{aligned}
\varphi(r) & =a \sin (\pi r), \\
\psi(r) & =b \sin (\pi r),
\end{aligned}
$$

with $a \in[0,2), b \in[0,1)$. We begin by the construction of some adaptive schemes as follows.

Let $I$ be a positive integer and let $h=1 / I$. Define the grid $x_{i}=i h, 0 \leq i \leq I$, and approximate the solution $(u, v)$ of (3.1)-(3.6) by the solution $\left(U_{h}^{(n)}, V_{h}^{(n)}\right)$ of the explicit scheme

$$
\begin{aligned}
\frac{U_{0}^{(n+1)}-U_{0}^{(n)}}{\Delta t_{n}}= & \varepsilon N \frac{2 U_{1}^{(n)}-2 U_{0}^{(n)}}{h^{2}}+\left(1-V_{0}^{(n)}\right)^{-p}, \\
\frac{V_{0}^{(n+1)}-V_{0}^{(n)}}{\Delta t_{n}}= & \varepsilon N \frac{2 V_{1}^{(n)}-2 V_{0}^{(n)}}{h^{2}}+\left(2-U_{0}^{(n)}\right)^{-q}, \\
\frac{U_{i}^{(n+1)}-U_{i}^{(n)}}{\Delta t_{n}}= & \varepsilon\left(\frac{U_{i+1}^{(n)}-2 U_{i}^{(n)}+U_{i-1}^{(n)}}{h^{2}}+\frac{(N-1)}{i h} \frac{U_{i+1}^{(n)}-U_{i-1}^{(n)}}{2 h}\right) \\
& +\left(1-V_{i}^{(n)}\right)^{-p}, \quad 1 \leq i \leq I-1, \\
\frac{V_{i}^{(n+1)}-V_{i}^{(n)}=}{\Delta t_{n}} & \varepsilon\left(\frac{V_{i+1}^{(n)}-2 V_{i}^{(n)}+V_{i-1}^{(n)}}{h^{2}}+\frac{(N-1)}{i h} \frac{V_{i+1}^{(n)}-V_{i-1}^{(n)}}{2 h}\right) \\
& +\left(2-U_{i}^{(n)}\right)^{-q}, \quad 1 \leq i \leq I-1, \\
& U_{I}^{(n)}=0, \quad V_{I}^{(n)}=0, \\
& U_{i}^{(0)}=a \sin (\pi i h), \quad 0 \leq i \leq I, \\
& V_{i}^{(0)}=b \sin (\pi i h), \quad 0 \leq i \leq I,
\end{aligned}
$$

where $n \geq 0$ and $U_{h}^{(n)}=\left(U_{0}^{(n)}, U_{1}^{(n)}, \ldots, U_{I}^{(n)}\right)^{T}, V_{h}^{(n)}=\left(V_{0}^{(n)}, V_{1}^{(n)}, \ldots, V_{I}^{(n)}\right)^{T}$.

In order to permit the discrete solution to reproduce the property of the continuous one when the time $t$ approaches the quenching time $T$, we need to adapt the size of the time step so that we take

$$
\Delta t_{n}=\min \left\{\frac{h^{2}}{2 N \varepsilon}, h^{2}\left(1-\left\|V_{h}^{(n)}\right\|_{\infty}\right)^{p+1}, h^{2}\left(2-\left\|U_{h}^{(n)}\right\|_{\infty}\right)^{q+1}\right\} .
$$


We also approximate the solution $(u, v)$ of (3.1)-(3.6) by the solution $\left(U_{h}^{(n)}, V_{h}^{(n)}\right)$ of the following implicit scheme:

$$
\begin{aligned}
& \frac{U_{0}^{(n+1)}-U_{0}^{(n)}}{\Delta t_{n}}= \varepsilon N \frac{2 U_{1}^{(n+1)}-2 U_{0}^{(n+1)}}{h^{2}}+\left(1-V_{0}^{(n)}\right)^{-p}, \\
& \frac{V_{0}^{(n+1)}-V_{0}^{(n)}}{\Delta t_{n}}= \varepsilon N \frac{2 V_{1}^{(n+1)}-2 V_{0}^{(n+1)}}{h^{2}}+\left(2-U_{0}^{(n)}\right)^{-q}, \\
& \frac{U_{i}^{(n+1)}-U_{i}^{(n)}}{\Delta t_{n}}= \varepsilon\left(\frac{U_{i+1}^{(n+1)}-2 U_{i}^{(n+1)}+U_{i-1}^{(n+1)}}{h^{2}}+\frac{(N-1)}{i h} \frac{U_{i+1}^{(n+1)}-U_{i-1}^{(n+1)}}{2 h}\right) \\
&+\left(1-V_{i}^{(n)}\right)^{-p}, \quad 1 \leq i \leq I-1, \\
& \frac{V_{i}^{(n+1)}-V_{i}^{(n)}}{\Delta t_{n}}= \varepsilon\left(\frac{V_{i+1}^{(n+1)}-2 V_{i}^{(n+1)}+V_{i-1}^{(n+1)}}{h^{2}}+\frac{(N-1)}{i h} \frac{V_{i+1}^{(n+1)}-V_{i-1}^{(n+1)}}{2 h}\right) \\
&+\left(2-U_{i}^{(n)}\right)^{-q}, \quad 1 \leq i \leq I-1, \\
& U_{I}^{(n+1)}=0, \quad V_{I}^{(n+1)}=0, \\
& U_{i}^{(0)}=a \sin (\pi i h), \quad 0 \leq i \leq I, \\
& V_{i}^{(0)}=b \sin (\pi i h), \quad 0 \leq i \leq I .
\end{aligned}
$$

Here, similarly to the case of the explicit scheme, we choose

$$
\Delta t_{n}=\min \left\{h^{2}\left(1-\left\|V_{h}^{(n)}\right\|_{\infty}\right)^{p+1}, h^{2}\left(2-\left\|U_{h}^{(n)}\right\|_{\infty}\right)^{q+1}\right\} .
$$

We note that

$$
\lim _{r \rightarrow 0} \frac{u_{r}(r, t)}{r}=u_{r r}(0, t) .
$$

Hence, if $t=0$, then we have

$$
u_{t}(0, t)=\varepsilon N u_{r r}(0, t)+(1-v(0, t))^{-p} .
$$

This observation has been used in the construction of our schemes when $i=0$. Let us notice that in the explicit scheme, the restriction on the time step ensures the nonnegativity of the discrete solution. For the implicit scheme, the existence and nonnegativity are also guaranteed by standard methods (see, e. g., [2]).

We need the following definition.

Definition 1. We say that the discrete solution $\left(U_{h}^{(n)}, V_{h}^{(n)}\right)$ of the explicit or implicit scheme quenches in a finite time if

$$
\left(\lim _{n \rightarrow+\infty}\left\|U_{h}^{(n)}\right\|_{\infty}, \lim _{n \rightarrow+\infty}\left\|V_{h}^{(n)}\right\|_{\infty}\right) \not 2(2,1),
$$


and the series $\sum_{n=0}^{+\infty} \Delta t_{n}$ converges. The quantity $\sum_{n=0}^{+\infty} \Delta t_{n}$ is called the numerical quenching time of the solution $\left(U_{h}^{(n)}, V_{h}^{(n)}\right)$.

In the following tables, in rows, we present the numerical quenching times, the numbers of iterations, CPU times and the orders of the approximations corresponding to meshes of $16,32,64,128,256$. We take for the numerical quenching time

$$
T^{n}=\sum_{j=0}^{n-1} \Delta t_{j},
$$

which is computed at the first time when

$$
\left|T^{n+1}-T^{n}\right| \leq 10^{-16} .
$$

The order $s$ of the method is computed according to the formula

$$
s=\frac{\log \left(\left(T_{4 h}-T_{2 h}\right) /\left(T_{2 h}-T_{h}\right)\right)}{\log (2)} .
$$

3.1. Numerical experiments for $p=1, q=1, a=0, b=0, N=2$

3.1.1. First case: $\varepsilon=\frac{1}{10}$

\begin{tabular}{|l|l|l|l|l|}
\hline$I$ & $T^{n}$ & $n$ & CPU time & $s$ \\
\hline 16 & 1.011490 & 8172 & - & - \\
\hline 32 & 1.010694 & 31009 & 1 & - \\
\hline 64 & 1.010360 & 118245 & 4 & 1.26 \\
\hline 128 & 1.010207 & 450669 & 30 & 1.13 \\
\hline 256 & 1.010135 & 1714170 & 230 & 1.10 \\
\hline
\end{tabular}

TABLE 1. Numerical quenching times, numbers of iterations, CPU times (seconds), and orders of the approximations obtained with the explicit Euler method.

\begin{tabular}{|l|l|l|l|l|}
\hline$I$ & $T^{n}$ & $n$ & CPU time & $s$ \\
\hline 16 & 1.015745 & 9004 & - & - \\
\hline 32 & 1.014494 & 34740 & 3 & - \\
\hline 64 & 1.014196 & 134302 & 16 & 2.08 \\
\hline 128 & 1.014124 & 518394 & 118 & 2.06 \\
\hline 256 & 1.014107 & 1996487 & 954 & 2.09 \\
\hline
\end{tabular}

TABLE 2. Numerical quenching times, numbers of iterations, CPU times (seconds) and orders of the approximations obtained with the implicit Euler method. 
3.1.2. Second case: $\varepsilon=\frac{1}{100}$

\begin{tabular}{|l|l|l|l|l|}
\hline$I$ & $T^{n}$ & $n$ & CPU time & $s$ \\
\hline 16 & 1.000142 & 8084 & - & - \\
\hline 32 & 1.000100 & 30610 & 1 & - \\
\hline 64 & 1.000088 & 116756 & 4 & 1.81 \\
\hline 128 & 1.000022 & 454329 & 31 & 2.46 \\
\hline 256 & 1.000006 & 1726227 & 231 & 2.05 \\
\hline
\end{tabular}

TABLE 3. Numerical quenching times, numbers of iterations, CPU times (seconds), and orders of the approximations obtained with the explicit Euler method.

\begin{tabular}{|l|l|l|l|l|}
\hline$I$ & $T^{n}$ & $n$ & CPU time & $s$ \\
\hline 16 & 1.000288 & 8087 & - & - \\
\hline 32 & 1.000134 & 30620 & 1 & - \\
\hline 64 & 1.000100 & 116520 & 14 & 2.18 \\
\hline 128 & 1.000093 & 443127 & 101 & 2.28 \\
\hline 256 & 1.000091 & 1681491 & 861 & 1.81 \\
\hline
\end{tabular}

TABLE 4. Numerical quenching times, numbers of iterations, CPU times (seconds) and orders of the approximations obtained with the implicit Euler method.

3.2. Numerical experiments for $p=1, q=1, a=0, b=0, N=2$

3.2.1. First case: $\varepsilon=\frac{1}{100}$

\begin{tabular}{|l|l|l|l|l|}
\hline$I$ & $T^{n}$ & $n$ & CPU time & $s$ \\
\hline 16 & 0.393420 & 11468 & - & - \\
\hline 32 & 0.393698 & 43641 & 1 & - \\
\hline 64 & 0.393784 & 166383 & 6 & 1.70 \\
\hline 128 & 0.393809 & 633406 & 45 & 1.78 \\
\hline 256 & 0.393817 & 2405445 & 338 & 1.65 \\
\hline
\end{tabular}

TABLE 5. Numerical quenching times, numbers of iterations, CPU times (seconds), and orders of the approximations obtained with the explicit Euler method. 


\begin{tabular}{|l|l|l|l|l|}
\hline$I$ & $T^{n}$ & $n$ & CPU time & $s$ \\
\hline 16 & 0.393440 & 11469 & - & - \\
\hline 32 & 0.393703 & 43641 & 2 & - \\
\hline 64 & 0.393785 & 166384 & 19 & 1.69 \\
\hline 128 & 0.393810 & 633407 & 153 & 1.72 \\
\hline 256 & 0.393817 & 2405445 & 1093 & 1.84 \\
\hline
\end{tabular}

TABLE 6. Numerical quenching times, numbers of iterations, CPU times (seconds) and orders of the approximations obtained with the implicit Euler method

3.2.2. Second case: $\varepsilon=\frac{1}{500}$

\begin{tabular}{|l|l|l|l|l|}
\hline$I$ & $T^{n}$ & $n$ & CPU time & $s$ \\
\hline 16 & 0.375615 & 11613 & - & - \\
\hline 32 & 0.375680 & 44079 & & - \\
\hline 64 & 0.375702 & 167676 & 6 & 1.57 \\
\hline 128 & 0.375709 & 636904 & 45 & 1.66 \\
\hline 256 & 0.375711 & 2413063 & 354 & 1.81 \\
\hline
\end{tabular}

TABLE 7. Numerical quenching times, numbers of iterations, CPU times (seconds), and orders of the approximations obtained with the explicit Euler method.

\begin{tabular}{|l|l|l|l|l|}
\hline$I$ & $T^{n}$ & $n$ & CPU time & $s$ \\
\hline 16 & 0.375619 & 11613 & - & - \\
\hline 32 & 0.375682 & 44079 & 3 & - \\
\hline 64 & 0.375702 & 167677 & 19 & 1.66 \\
\hline 128 & 0.375709 & 636904 & 150 & 1.52 \\
\hline 256 & 0.375711 & 2413063 & 1120 & 1.81 \\
\hline
\end{tabular}

TABLE 8. Numerical quenching times, numbers of iterations, CPU times (seconds) and orders of the approximations obtained with the implicit Euler method.

Remark. If we consider the problem (3.1)-(3.6) in the case where the initial data is null and $p=1, q=1$, it is not difficult to see that the quenching time of the solution of the differential system defined in (1.11)-(1.14) equals one. We observe from Tables 1-4 that when $\varepsilon$ diminishes, then the numerical quenching time tends to one. This result has been proved in Theorem 1 .

When the initial data $(\varphi(r), \psi(r))$ are such that $\varphi(r)=\frac{1}{2} \sin (\pi r)$ and $\psi(r)=$ $\frac{1}{2} \sin (\pi r)$, and $p=1, q=1$, then we see that the quenching time of the solution 
of the differential system defined in (1.11)-(1.14) equals 0.375 . We observe from Tables 5-8 that when $\varepsilon$ diminishes, then the numerical quenching time decays to 0.375 . This result has been established in Theorem 2 .

\section{REFERENCES}

[1] T. K. Boni, "On quenching of solutions for some semilinear parabolic equations of second order," Bull. Belg. Math. Soc. Simon Stevin, vol. 7, no. 1, pp. 73-95, 2000. [Online]. Available: http://projecteuclid.org/getRecord?id=euclid.bbms/1103055721

[2] T. K. Boni, "Extinction for discretizations of some semilinear parabolic equations," $C . R$. Acad. Sci. Paris Sér. I Math., vol. 333, no. 8, pp. 795-800, 2001. [Online]. Available: http://dx.doi.org/10.1016/S0764-4442(01)02078-X

[3] C. Y. Chan and C. S. Chen, "Critical lengths for global existence of solutions for coupled semilinear singular parabolic problems," Quart. Appl. Math., vol. 47, no. 4, pp. 661-671, 1989.

[4] C. Y. Chan and D. T. Fung, "Quenching for coupled semilinear reaction-diffusion problems," Nonlinear Anal., vol. 21, no. 2, pp. 143-152, 1993. [Online]. Available: http://dx.doi.org/10.1016/0362-546X(93)90044-S

[5] Q. Dai and Y. Gu, "Quenching phenomena for systems of semilinear parabolic equations. I," Systems Sci. Math. Sci., vol. 10, no. 4, pp. 361-371, 1997.

[6] K. Deng, "Dynamical behavior of solutions of a semilinear heat equation with nonlocal singularity," SIAM J. Math. Anal., vol. 26, no. 1, pp. 98-111, 1995. [Online]. Available: http://dx.doi.org/10.1137/S0036141091223881

[7] K. Deng and C. A. Roberts, "Quenching for a diffusive equation with a concentrated singularity," Differential Integral Equations, vol. 10, no. 2, pp. 369-379, 1997.

[8] A. Friedman and A. A. Lacey, "The blow-up time for solutions of nonlinear heat equations with small diffusion," SIAM J. Math. Anal., vol. 18, no. 3, pp. 711-721, 1987. [Online]. Available: http://dx.doi.org/10.1137/0518054

[9] H. A. Levine, "Quenching, nonquenching, and beyond quenching for solution of some parabolic equations," Ann. Mat. Pura Appl. (4), vol. 155, pp. 243-260, 1989. [Online]. Available: http://dx.doi.org/10.1007/BF01765943

[10] D. Nabongo and T. K. Boni, "Quenching time of solutions for some nonlinear parabolic equations," An. Ştiinţ. Univ. “Ovidius” Constanţa Ser. Mat., vol. 16, no. 1, pp. 91-106, 2008.

\section{Authors' addresses}

Theodore K. Boni

Institut National Polytechnique Houphouet-Boigny de Yamoussoukro, Département de Mathématiques et Informatiques, BP 1093 Yamoussoukro, Côte d'Ivoire

E-mail address: theokboni@yahoo.fr

Halima Nachid

Université d'Abobo-Adjame, UFR-SFA, Département de Mathématiques et Informatiques, 16 BP 372, Abidjan 16, Côte d'Ivoire

E-mail address: nachidhalima@yahoo.fr

Diabate Nabongo

Université d'Abobo-Adjame, UFR-SFA, Département de Mathématiques et Informatiques, 16 BP 372, Abidjan 16, Côte d'Ivoire

E-mail address: nabongo_diabate@yahoo.fr 